\title{
COLLECTIVE EFFECTS IN SHOCK PROPAGATION THROUGH A CLUMPY MEDIUM
}

\author{
M.L. Norman ${ }^{*}$, J.R. Dickel, M. Livio and Y.-H. Chu \\ University of Illinois at Urbana-Champaign, \\ Urbana, IL 61801
}

\begin{abstract}
A numerical simulation of shock propagation in a clumpy medium with a weak magnetic field is presented which illustrates a number of dynamical processes of potential importance for explaining spectral line width and radio polarization measurements in supernova remnants.
\end{abstract}

Introduction: There is considerable evidence to support the suggestion by McKee and Cowie (1975) that the blast wave in an expanding supernova remnant (SNR) encounters density irregularities in the surrounding medium. Dense clouds will get swept up and eventually evaporate in their hotter shocked surroundings. The data include both optical and radio images showing numerous small-scale features with sizes ranging down to less than the instrumental resolution. Recently Shull (1983a,b) has found spectral line widths of over $100-200 \mathrm{~km} / \mathrm{s}$ in sections of SNRs N49 and N63A in the Large Magellanic Cloud indicating a large velocity dispersion within a single cloud; this might represent random motions of small cloudlets within the larger feature. Another prominent feature of SNR is an apparent net radial stretching of their magnetic field lines which could be caused by the distortion and wrapping around of the edges of clouds encountered by the outward moving shock. Theoretical estimates of the energy and mass involved in the interactions and evaporation of clouds give parameters which match the observed expansion (Cowie, McKee and Ostriker 1981). Finally, a 1-dimensional hydrodynamic model has been used to show that the observed shell thickness and radial brightness variations in young SNR can be explained by interactions with a number of little clumps (Dickel, Eilek and Jones 1987).

The true situation will involve the interactions of the initial shock with a collection of randomly placed clumps. The result will be a complex pattern of colliding shocks which have been both reflected and transmitted at different speeds through the different densities encountered. The process will distort the density, pressure, magnetic field structure, and other parameters of the clump and interclump regions. To assess the resultant structures and confirm the viability of such a phenomenon to actually explain the observations we have utilized a 2-dimensional magnetohydrodynamics code to follow all the physical variables as the shock propagates through the clumpy medium.

Numerical Methodology: The initial conditions for the simulation consist of a uniform background gas to which a number of gaussian-shaped dense clumps are added, randomly distributed within the two-dimensional problem domain $(0 \leq X \leq 600 ; 0 \leq Y \leq 400)$. The clumps have a maximum density of twenty times the interclump gas density, but are in pressure balance with the interclump gas by virtue of their lower temperature. In addition, a uniform weak magnetic field is initialized oriented parallel to the $X$ axis. A Mach number $=10$ planar shock is injected along the $Y=0$ boundary at $t=0$ and driven in time through an appropriate choice of inflow boundary conditions. An outflow boundary condition is used at $Y=400$ so that the shock may exit the problem domain. Periodic boundary conditions are applied at $X=0$ and $X=600$. $A \gamma=5 / 3$ ideal gas equation of state is assumed for both the clump and interclump gas. As we assume radiative cooling to be unimportant, the results of the simulation are scale invariant and are wholly determined by the dimensionless parameters of the problem.

* also, National Center for Supercomputing Applications 
The equations of ideal magnetohydrodynamics are advanced in time subject to these initial and boundary conditions using the ZEUS code under development at the National Center for Supercomputing Applications, University of Illinois, Urbana-Champaign. ZEUS is an outgrowth of an earlier, purely hydrodynamical code (Norman and Winkler 1986). The magnetic vector potential which describes the magnetic field is evolved along with the conservation laws for mass, momentum and energy in an inviscid gas, taking into account the JxB Lorentz force on the fluid trajectories (Clarke, Norman and Burns 1986). ZEUS is an Eulerian finite-difference code which employs the second order-accurate monotonic upwind advection scheme of Van Leer (1977) for all field variables except for the vector potential, which is advected using the third order-accurate PPM advection scheme of Colella and Woodward (1984). This is necessary since the magnetic field strength, which is given by the curl of the vector potential, is required to be second order-accurate in keeping with the other field variables. Shock waves are treated using the Von Neumann-Richtmyer artificial viscosity. In general, the dynamical equations are time-differenced in an explicit fashion, the exception being the use of a time-implicit energy equation for flows with strong cooling. ZEUS has been extensively tested against a variety of flow problems where the solution is either known analytically or has been determined experimentally. A grid of $300 \times 200$ uniform zones is used for the present simulation, which required approximately 2 hours of cpu time on a CRAY XMP.

Results: The shock front corrugates as it propagates through regions of different densities and sound speeds introduced by the clumps (Fig. 1a). When the incident shock wave encounters a clump, it is transmitted more slowly than in the interclump gas, producing the dimples seen in Fig. 1b. A reflected shock wave is also produced upstream of each clump. These merge to form the intersecting bow shocks seen in Fig. 1b. The effect of the transmitted shock wave on a clump is to flatten and compress it while imparting to it a velocity normal to the local shock surface. By virtue of the shock front corrugations, a range of transverse velocities are imparted since it can happen that an individual clump is struck at an angle to the overall direction of shock propagation.

a)

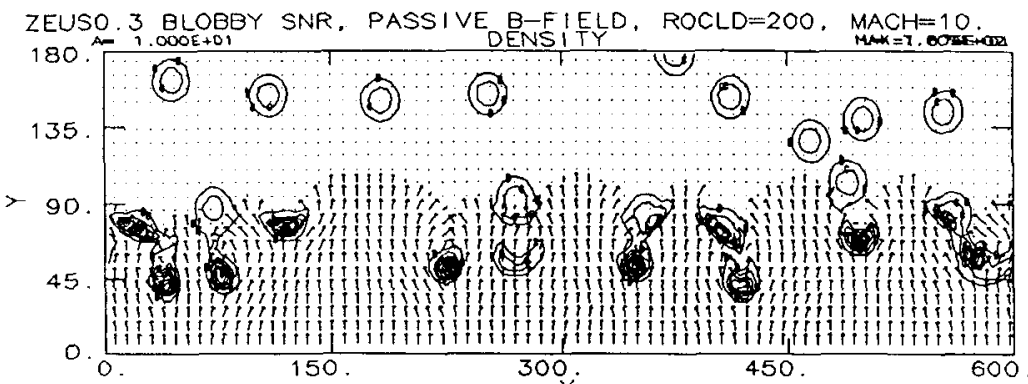

b)

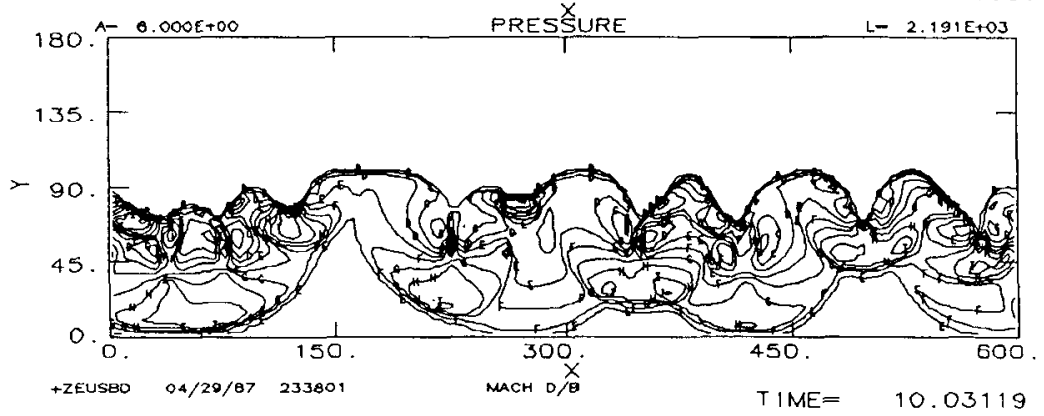

Fig. 1 Density contours and velocity vectors (a) and pressure contours (b) showing the corrugation of the shock front as it encounters the clumps (small circles in Fig. 1a). 


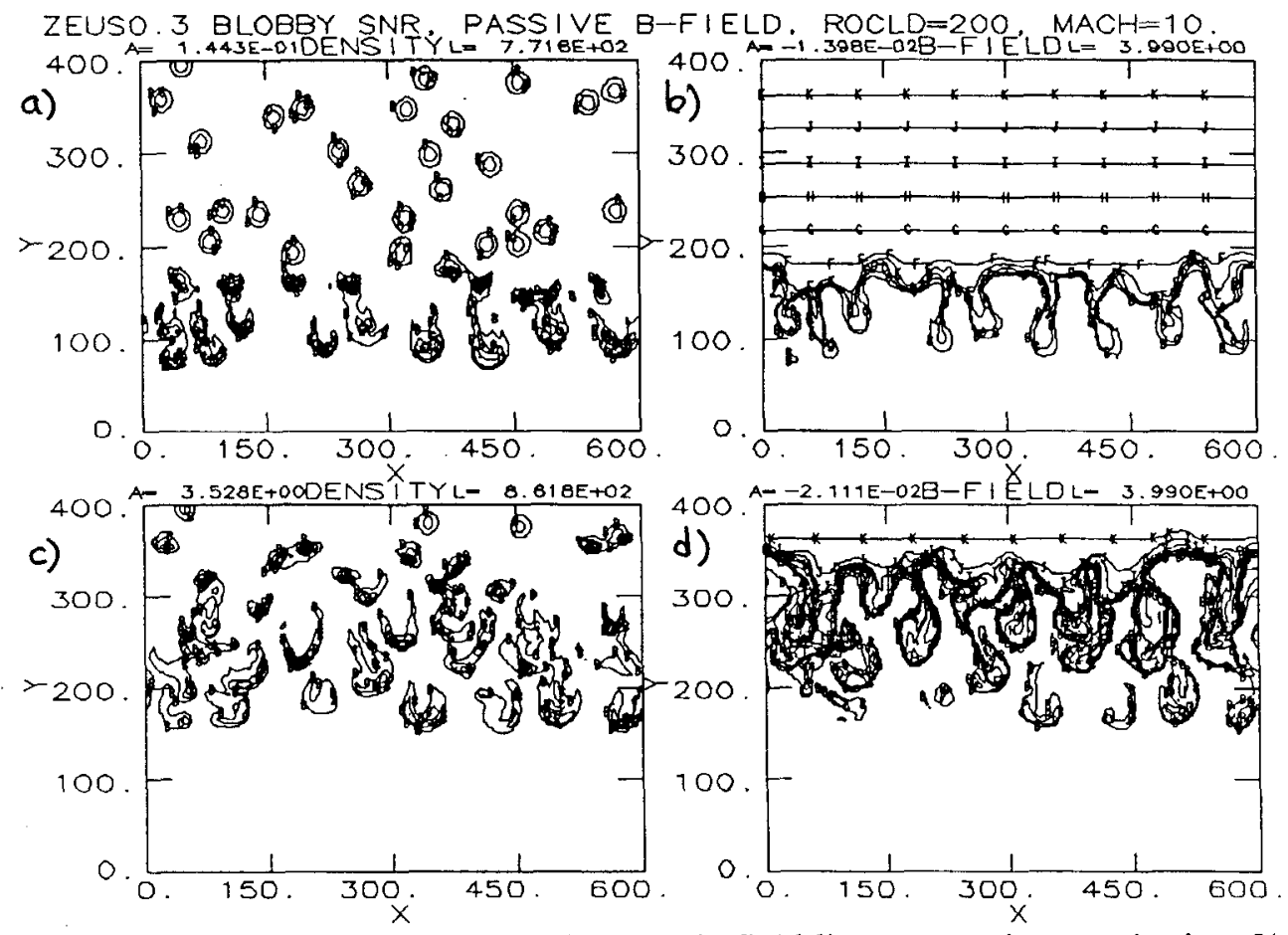

Fig. 2 Distribution of gas density and magnetic field lines at two instants in time [(a,b) $\mathrm{t}=20 ;(\mathrm{c}, \mathrm{d}) \mathrm{t}=40$ ], showing the development of horseshoe clump geometry and vertical stretching of magnetic field lines.

With time, the shocked clumps assume a characteristic horseshoe shape, as shown in Fig. 2a,c. This shape is produced by the cross-stream variation of the pressure difference between the upwind and lea sides of the clump. Magnetic field lines, which are anchored to the clumps via flux freezing, are stretched downstream in the interclump gas, as shown in Figs. 2b,d. Downstream of each clump, the magnetic field geometry is that of oppositely directed field lines -- ideal conditions for magnetic reconnection. Although our code assumes zero resistivity, numerical truncation errors introduce a grid resistivity which snaps magnetic field lines on the resolution scale of the calculation. As each clump is resolved by thirty or more zones in each direction, the large scale magnetic field geometry is faithfully simulated, however.

By the time the shock wave has crossed the problem domain, it has interacted with each clump, setting them all in motion. An individual clump will be accelerated in a direction determined by the local shock normal, which in turn is determined by the location of neighboring clumps. As a result of this shock-mediated collective interaction of clumps, a range of longitudinal and transverse velocities are produced. To quantify this effect, theoretical line profiles were calculated by binning in velocity space the square of the gas density moving at a particular longitudinal (parallel to the overall shock propagation direction) or transverse speed. These two distributions were then normalized and plotted in Fig. 3. As can be seen, each distribution consists of a narrow central core and broad wings. Notice that the velocity widths in both the core and wings are comparable between the two orientations, indicating an effective thermalization of shock directed kinetic energy into clump random kinetic energy. 
LONGITUDINAL PROF ILE

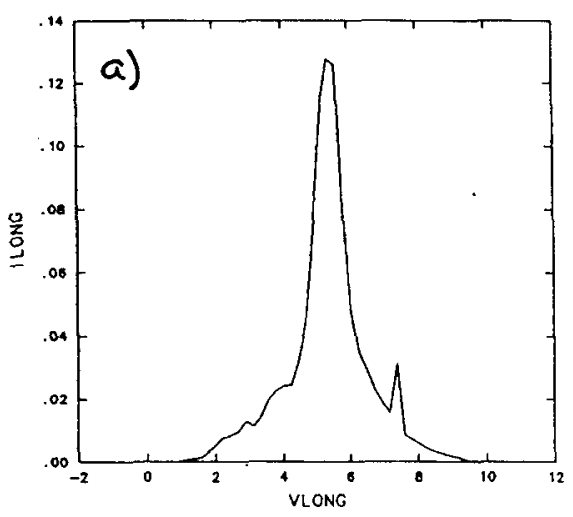

TRANSVERSE PROFILE

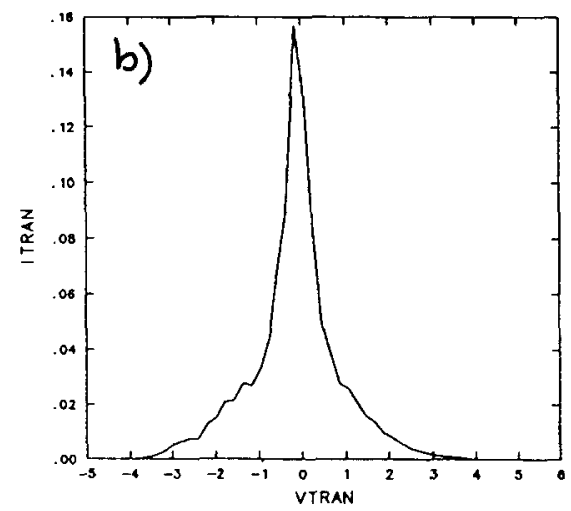

Fig. 3 Theoretical normalized line profiles at two viewing angles to the initial shock propagation direction, (a) parallel, (b) perpendicular. $\mathrm{V}_{\text {long }}$ and $\mathrm{V}_{\text {tran }}$ are in units of the interclump soundspeed $(=1)$; the shock velocity $=10$. Note the narrow core and broad wings have comparable velocity widths in both projections, indicating an effective thermalization of shock directed kinetic energy into clump random kinetic energy via collective effects.

Conclusions: Line widths comparable to the speed of the SNR blastwave can be produced via a shock-mediated collective interaction of dense clumps provided that their filling factor is sufficiently high. The assumed $11 \%$ filling factor in this $2-\mathrm{D}$ simulation would translate into a $3 \%$ filling factor in 3-D, which is not unrealistic. Theoretical line profiles consist of a narrow central core surrounded by broad wings which, in this simulation, are quite insensitive to viewing angle. We would not expect this to be the case at lower clump filling factors. Weak magnetic field lines permeating the unshocked clumpy medium will be stretched in the direction of shock propagation, which could account for the net radial polarization seen at centimeter wavelengths in many SNRs (e.g., Cas A).

This work was supported by an internal research grant at the National Center for Supercomputing Applications, University of Illinois at Urbana-Champaign.

\section{References:}

Clarke, D.A., Norman, M.L. and Burns, J.O. 1986, Ap.J. Lett., 311, L63.

Colella, P. and Woodward, P.R. 1984, J. Comp. Phys., 54, 115.

Cowie, L.L., McKee, C.F. and Ostriker, J.P. 1981, Ap.J., 247, 908.

Dickel, J.R., Eilek, J.A., and Jones, E.M. 1987, this volume.

McKee, C.F. and Cowie, L.L. 1975, Ap.J., 195, 715.

Norman, M.L. and Winkler, K.-H.A. 1986, in Astrophysical Radiation Hydrodynamics,

NATO ASI C188, eds. K.-H.A. Winkler and M.L. Norman, (Reidel:Dordrecht), 187.

Shull, P. 1983a, Ap.J., 275, 611. 1983b, Ap.J., 275, 592.

VanLeer, B. 1977, J. Comp. Phys., 23, 276. 